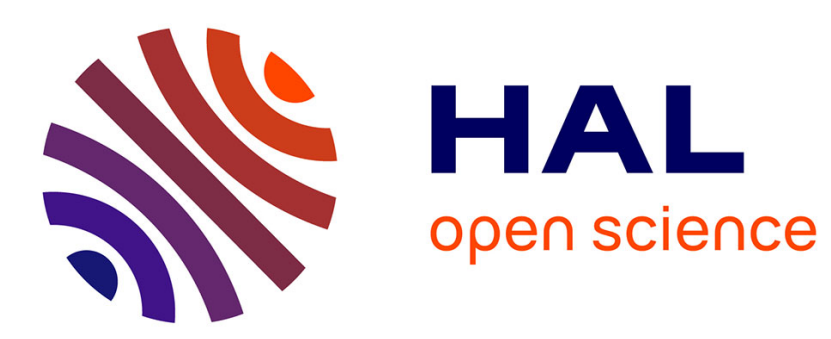

\title{
State-to-state inelastic rate coefficients of phosphine in collision with He atlow to moderate temperature
}

Ayda Badri, Faouzi Najar, Cheikh Bop, Nejm-Eddine Jaidane, Majdi Hochlaf

\section{To cite this version:}

Ayda Badri, Faouzi Najar, Cheikh Bop, Nejm-Eddine Jaidane, Majdi Hochlaf. State-to-state inelastic rate coefficients of phosphine in collision with He atlow to moderate temperature. Monthly Notices of the Royal Astronomical Society, 2020, 499 (2), pp.1578-1586. 10.1093/mnras/staa2611 . hal03078779

\section{HAL Id: hal-03078779 \\ https://hal.science/hal-03078779}

Submitted on 16 Dec 2020

HAL is a multi-disciplinary open access archive for the deposit and dissemination of scientific research documents, whether they are published or not. The documents may come from teaching and research institutions in France or abroad, or from public or private research centers.
L'archive ouverte pluridisciplinaire $\mathbf{H A L}$, est destinée au dépôt et à la diffusion de documents scientifiques de niveau recherche, publiés ou non, émanant des établissements d'enseignement et de recherche français ou étrangers, des laboratoires publics ou privés. 


\title{
State-to-state inelastic rate coefficients of phosphine in collision with He at low to moderate temperature
}

\author{
Ayda Badri, ${ }^{1,2}$ Faouzi Najar, ${ }^{1}$ Cheikh T. Bop, ${ }^{3,4}$ Nejm-Eddine Jaidane ${ }^{1}$ and Majdi Hochlaf ${ }^{\circledR 2 \star ~}$ \\ ${ }^{1}$ Laboratoire de Spectroscopie Atomique, Moléculaire et Applications LSAMA, Université de Tunis El Manar, Tunis, Tunisia \\ ${ }^{2}$ Université Gustave Eiffel, COSYS/LISIS, 5 Bd Descartes, 77454 Champs sur Marne, France \\ ${ }^{3}$ Laboratory of Atoms Lasers, Department of Physics, Faculty of Sciences and Techniques, University Cheikh Anta Diop of Dakar, Dakar 5005, Senegal \\ ${ }^{4}$ LOMC-UMR 6294 CNRS, Université du havre, 25 rue Philippe Lebon, BP 1123, F-76063 Le Havre, France
}

Accepted 2020 August 20. Received 2020 August 19; in original form 2020 May 20

\begin{abstract}
Several phosphorus-bearing molecules, such as the phosphine of interest here, have been detected in astrophysical media. With the aim of satisfying the precision required by the astrophysical community, we report the rate coefficients of $\mathrm{PH}_{3}$ in collision with helium from low to moderate temperature. To this end, we constructed the first three-dimensional potential energy surface (3D-PES) of the $\mathrm{PH}_{3}-\mathrm{He}$ van der Waals complex, which governs the nuclear motions. The 3D-PES was worked out by means of the standard coupled cluster with single, double and non-iterative triple excitation approach, in conjunction with the aug-cc-pVQZ basis set and complemented by mid bond functions. This 3D-PES presents a well of $34.92 \mathrm{~cm}^{-1}$ at $\{R, \theta, \Phi\}$ $=\left\{5.76 a_{0}, 90^{\circ}, 60^{\circ}\right\}$. Afterwards, we incorporated this 3D-PES into time-independent close-coupling quantum dynamical computations to derive the inelastic cross-sections of rotational excitation of (ortho-) para- $\mathrm{PH}_{3}$ after collision with He up to (1000) $500 \mathrm{~cm}^{-1}$. Subsequently, we evaluated the rate coefficients for temperatures up to (100 K) $50 \mathrm{~K}$ populating the (41) 42 low-lying rotational levels of (ortho-) para- $\mathrm{PH}_{3}$. These data were derived by averaging the cross-sections thermally over the Maxwell-Boltzmann velocity distribution. No general propensity rules are found. We also performed a comparison with the rates for $\mathrm{NH}_{3}-\mathrm{He}$. Differences are observed that invalidate the use of $\mathrm{NH}_{3}$ rates for deducing accurate abundances of phosphine in cold astrophysical media. Our results should be of great help in determining accurate $\mathrm{PH}_{3}$ abundances and, more generally, constraining the interstellar $\mathrm{PH}_{3}$ chemistry better.
\end{abstract}

Key words: ISM : abundances-ISM: molecules.

\section{INTRODUCTION}

The second-row elements of the periodic table are present in the interstellar medium (ISM) with relatively low cosmic standards. Among them, phosphorous (known also as a biogenic element) is one of the less abundant. In fact, only one out of the 23 known nuclides of phosphorous is stable $\left({ }^{31} \mathrm{P}\right)$, with a binding energy of $\sim 8.4 \mathrm{MeV}$ (Maciá 2005). However, its formation throughout thermonuclear fusion requires temperatures above $10^{9} \mathrm{~K}$. Indeed, the barrier of electrostatic repulsion among the colliding nuclei related to a nuclear charge of $Z=15$ (i.e. ${ }^{31} \mathrm{P}$ formation) imposes a great amount of energy. These items let us expect that thermonuclear reactions leading to the formation of phosphorous can take place only in the cores of massive stars of size 15-100 $\mathrm{M}_{\odot}$ (Arnett \& Arnett 1996; Maciá 2005). For instance, the early origin of the ${ }^{31} \mathrm{P}$ isotope occurred towards the inner zone of stars sufficiently massive to undergo successive carbon- and neon-burning at approximately $T=3 \times 10^{9} \mathrm{~K}$ (Woosley 2002). Once produced in the cores of massive stars, this biogenic element is likely carried towards the ISM by supernova explosions (by the birth of planetary nebulae) in the case of very high-mass stars (of relatively high-mass stars) and stellar

^E-mail: hochlaf@univ-mlv.fr winds. The stable phosphorus thus produced and liberated in the ISM would be ready to react with available atoms, leading to the formation of interstellar P-bearing compounds. Among them, phosphine, $\mathrm{PH}_{3}$, of interest in the present work, is expected to be the main reservoir of phosphorous in space. Indeed, $\mathrm{PH}_{3}$ has been observed in various astronomical environments over these last four decades (Moreno, Marten \& Lellouch 2009; Weisstein \& Serabyn 1996; Larson 1977; Bregman \& Rank 1975). For instance, phosphine was definitely detected in several extraterrestrial environments. In the ISM, it was observed in the circumstellar envelope of the carbon star IRC +10216 (Agúndez et al. 2008; Tenenbaum \& Ziurys 2008a). It is supposed to be formed on interstellar grains via successive hydrogenation of atomic phosphorus (Jiménez-Serra et al. 2018) and then desorbs to the gas phase. Phosphine was also identified in the atmospheres of Jupiter and Saturn (Ridgway, Wallace \& Smith 1976; Larson et al. 1980). Thanks to the Herschel Spectral and Photometric Imaging Receiver (SPIRE), high-resolution far-IR observations of Saturn's atmosphere allowed us to determine the vertical distribution of $\mathrm{PH}_{3}$. Moreover, this molecule is most likely present in the atmospheres of brown dwarfs or giant extrasolar planets (Sharp \& Burrows 2007). Furthermore, it is connected to the origin of the phosphorus signal in comet 67P/Churyumov-Gerasimenko (Altwegg 2016).

In addition to $\mathrm{PH}_{3}$, five other $\mathrm{P}$-containing molecules, namely $\mathrm{PN}$, PO, CP, HCP, CCP and NCCP, have been successfully detected in 
interstellar and circumstellar regions (Turner \& Bally 1987; Ziurys 1987; Guélin et al. 1990; Turner et al. 1990; Agúndez, Cernicharo \& Guélin 2007; Halfen, Clouthier \& Ziurys 2008; Milam et al. 2008). In terms of abundances, $\mathrm{PH}_{3}$ and $\mathrm{HCP}$, which are the dominant $\mathrm{P}$-bearers in IRC+10216, hold only 2 (Agúndez et al. 2014) and 5 percent (Agúndez et al. 2007) of atomic phosphorus, respectively. For other P-bearing compounds, the abundance ratio with respect to $\mathrm{P}$ drops to less than 0.001 (e.g. PN/P is about $5 \times 10^{-4}$ : Turner et al. 1990). Otherwise, gas-phase molecules in the envelopes of evolved stars lock a greater amount of phosphorus than in IRC+10216. Typically, in the carbon-rich envelope CRL 2668, $\mathrm{HCP}$ along with $\mathrm{PH}_{3}$ holds about half the phosphorus (Milam et al. 2008; Tenenbaum \& Ziurys $2008 \mathrm{~b}$ ), while a quarter of almost all the available phosphorus is in the form of PN and PO towards the oxygen-rich supergiant star VYCMa (Tenenbaum, Woolf \& Ziurys 2007; Milam et al. 2008) or towards the star IK Tau (De Beck et al. 2013). These items are in concordance with the hypothesis of Turner et al. (1990), which was based on the weak presence of P-bearers in interstellar clouds, suggesting that phosphorus is depleted in dust grains. Nevertheless, chemical models for identification of the formation pathways of Pbearing molecules in astrophysical media suffer from the lack of accurate abundances for these species to assess the initial elemental abundance of phosphorus, which is connected with the depletion level of P (Chantzos et al. 2020).

For the identification of molecules in astrophysical media, one needs accurate determinations of line positions and abundances. For $\mathrm{PH}_{3}$, while its rotation-vibration spectrum is well-established theoretically and experimentally (Sousa-Silva et al. 2015), its abundance is not. Indeed, its abundance is estimated using the collision rates of isovalent ammonia. Therefore, accurately determining the abundances of phosphine may be a clue to understanding its formation and phosphorus chemistry in space.

In order to satisfy astrophysical precision when modelling the abundance of interstellar species observed in media where local thermodynamic equilibrium (LTE) conditions are not reached, one relies on the large velocity gradient approach (LVG). Such a procedure requires preliminary calculations of rate coefficients induced by collision with the most abundant species $\left(\mathrm{H}_{2}, \mathrm{H}\right.$ and $\left.\mathrm{He}\right)$, as well as Einstein coefficients. These latter are often known, while collisional rates are not. In the case of a lack of such data for a newly observed molecule, astrophysicists usually deduce its collisional rates from those of isoelectronic species. Indeed, this approximation has been adopted for some molecules (Barlow et al. 2013; Cernicharo et al. 2018) and its limits were pointed out thereafter (Bop et al. 2016). In the case of phosphine, the approximation made by Agúndez et al. (2014), who used the rate coefficients of $\mathrm{NH}_{3}$ (Danby et al. 1988; Machin \& Roueff 2005), could be a priori more reliable. Nevertheless, this hypothesis deserves to be checked by computing the actual $\mathrm{PH}_{3}$ rate coefficients.

In this article, we investigate the rotational (de-)excitation of $\mathrm{PH}_{3}$ colliding with $\mathrm{He}$. Even if $\mathrm{He}$ is not the dominant collision partner in the ISM, the rate coefficients it yields may be used to evaluate those that would be obtained using para- $\mathrm{H}_{2}(j=0)$ as a projectile. Indeed, this approximation takes its suitability from the fact that para- $\mathrm{H}_{2}(j=0)$ is spherically symmetric and contains two valence electrons as helium. In addition, using helium as a projectile may be useful in the experimental framework for the interpretation of measured broadening parameters (Pickett, Poynter \& Cohen 1981; Levy, Lacome \& Tarrago 1994; Salem et al. 2005). Therefore, we generated the first 3D potential energy surface (3D-PES) of the $\mathrm{PH}_{3}-$ He van der Waals complex using the coupled cluster approach. This 3D-PES is mapped in Jacobi coordinates as defined in Fig. 1. Then

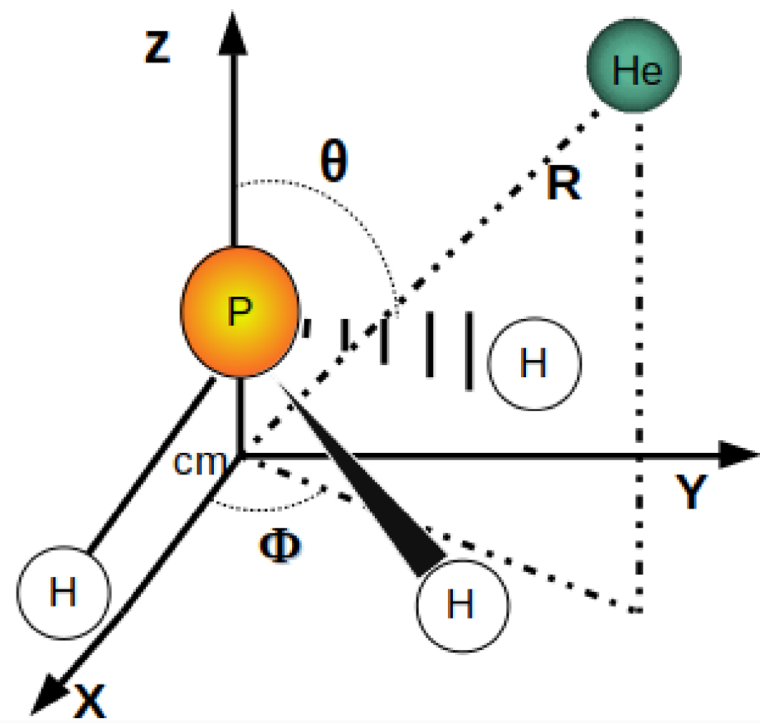

Figure 1. Description of the body-fixed coordinate system used in the calculations; $\mathrm{cm}$ stands for the centre of the $\mathrm{PH}_{3}$ molecule.

an analytic expansion of the 3D-PES was obtained by fitting the computed energies. Later on, this 3D-PES is incorporated into timeindependent quantum nuclear motion treatments to obtain the collision cross-sections and the collision rates after thermal averaging. Both ortho- and para- $\mathrm{PH}_{3}$ were considered. As said above, the present set of data can be used to estimate the abundances of phosphine better and to model phosphorus chemistry in astrophysical media.

\section{GENERATION OF THE 3D POTENTIAL ENERGY SURFACE OF $\mathrm{PH}_{3}-\mathrm{HE}$}

\subsection{Potential energy surface}

The system of interest $\left(\mathrm{PH}_{3}-\mathrm{He}\right)$ results from the interaction between a symmetric top molecule and a structureless atom in their ground electronic states, $\mathrm{PH}_{3}\left(\tilde{X}^{1} A_{1}\right)$ and $\mathrm{He}\left({ }^{1} \mathrm{~S}\right)$ respectively. The phosphine monomer was held fixed in its spatial configuration, as recommended by Faure et al. (2005), i.e. using the geometries of the vibrationless state. Typically, the distance between phosphorus $(\mathrm{P})$ and each hydrogen $(\mathrm{H})$ atom was set to $r_{\mathrm{PH}}=1.421 \AA$ and the angle $\widehat{\mathrm{HPH}}=93.3^{\circ}$ (Herzberg 1966).

Taking into account the relatively large electron number of the titled system, it is desirable to reduce the number of degrees of freedom in the construction of the interaction potential, while keeping its efficiency in yielding accurate dynamic results. For the inelastic scattering of $\mathrm{NH}_{3}$ due to He impact, neglecting umbrella inversion motion in the potential construction - as a model treatment - has led to errors in the amplitudes of the resonance peaks of less than 10 per cent relative to those obtained via a more elaborate treatment ,where this motion was considered fully in the interacting PES (Gubbels et al. 2012). These errors in the amplitudes are rather small. At first glance, one can omit this motion. Consequently, the complex can be described in the $\mathrm{PH}_{3}$ body-fixed (Jacobi) coordinate system using three parameters: (i) the distance $(R)$ between helium and the centre of mass of phosphine, (ii) the angle $(\theta)$, which stands for the rotation of He relative to the $z$-axis, and (iii) the rotation of $\mathrm{PH}_{3}(\Phi)$ with respect to the projection of the collision axis on the $x y$-plane (see Fig. 1). Therefore, the 3D-PES was constructed using 42 values of $R$ ranging from 4 to $40 a_{0}, 19 \mathrm{He}$ orientations 
(a)

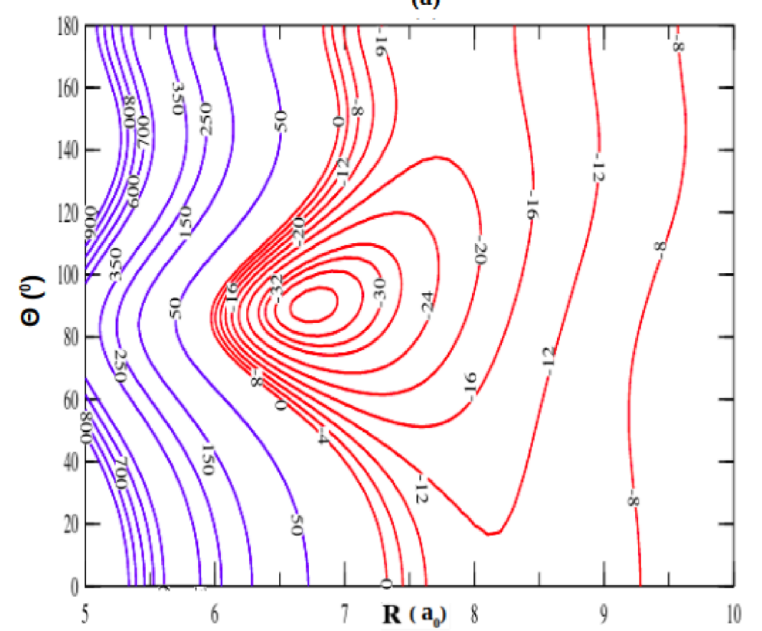

(b)

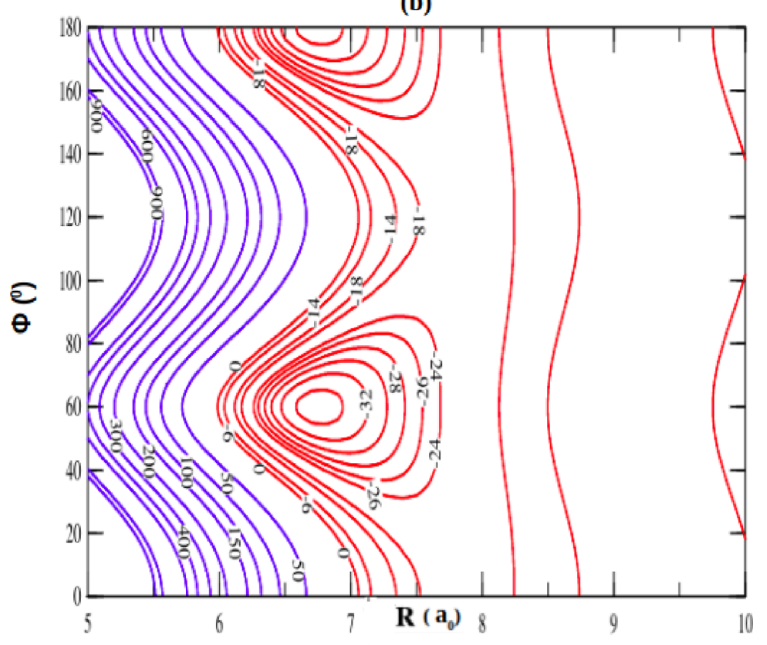

(c)

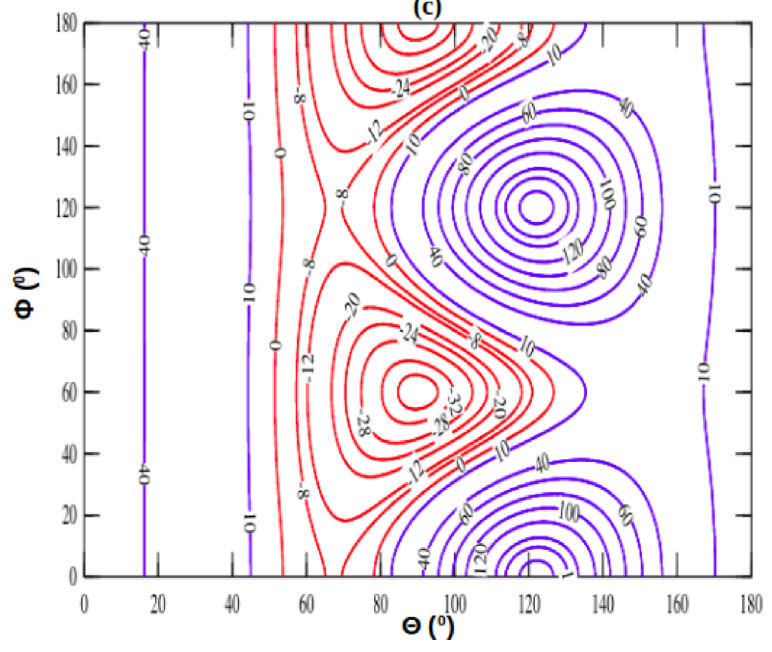

Figure 2. Two-dimensional contour plots of the three-dimensional potential energy surface of the $\mathrm{PH}_{3}-\mathrm{He}$ van der Waals complex. Panels (a), (b) and (c) depict respectively the 3D-PES as a function of $\theta$ and $\mathrm{R}$ at $\Phi=60^{\circ}, \Phi$ and $\mathrm{R}$ at $\theta=90^{\circ}$, and $\theta$ and $\Phi$ at $\mathrm{R}=6.75 a_{0}$. For each panel, the blue (red) contours represent the positive (negative) parts of the potential (in units of $\mathrm{cm}^{-1}$ ). The reference energy is taken as that of the separated monomers.

$\left(0^{\circ} \leq \theta \leq 180^{\circ}\right)$ and seven $\Phi$ angles in the range $\left[0-60^{\circ}\right]$. All these parameters have led to a set of 5586 non-redundant nuclear configurations for which we performed $a b$ initio electronic structure computations using the MOLPRO molecular package (Werner et al. 2010). Also, we used symmetry considerations to deduce the energies of the nuclear geometries not covered by our grid of points.

The 3D-PES was calculated using the coupled cluster with single, double and non-iterative triple excitations $(\operatorname{CCSD}(\mathrm{T}))$ method (Watts, Gauss \& Bartlett 1993) in conjunction with the augmentedconsistent correlation-polarized valence triple zeta (aug-cc-pVQZ) Gaussian basis sets of Dunning and co-workers (Dunning 1989), supplemented by the bond functions (bf) of Cybulski \& Toczyłowski (1999). These latter $(3 \mathrm{~s} 3 \mathrm{p} 2 \mathrm{~d} 2 \mathrm{f} 1 \mathrm{~g})$ were placed at mid-distance between the two monomers. This level of theory will be denoted thereafter as $\operatorname{CCSD}(\mathrm{T}) /$ aug-cc-pVQZ+bf. In addition, we used the counterpoise procedure of Boys \& Bernardi (1970) to correct for the basis set superposition errors (BSSE) using the following expression:

$V(R, \theta, \Phi)=E_{\mathrm{PH}_{3}-\mathrm{He}}(R, \theta, \Phi)-E_{\mathrm{PH}_{3}}(R, \theta, \Phi)$

$$
-E_{\mathrm{He}}(R, \theta, \Phi) \text {. }
$$

The accuracy of the interaction potentials generated using the methodology elaborated above has been assessed in our previous works (Najar et al. 2017; El Hanini et al. 2019).

We display in Fig. 2 the two-dimensional cuts of the 3D-PES of the $\mathrm{PH}_{3}-\mathrm{He}$ weakly bound system as a function of two Jacobi coordinates, whereas the third one was kept fixed at its equilibrium value in the $\mathrm{PH}_{3}-\mathrm{He}$ minimum. Indeed, we give the $3 \mathrm{D}-\mathrm{PES}$ as a function of $\theta$ and $R$ at $\Phi=60^{\circ}, \Phi$ and $R$ at $\theta=90^{\circ}$ and $\theta$ and $\Phi$ for $R=6.75 \mathrm{bohr}$. The positive parts of the 3D-PES are in blue and the negative ones are in red, where the reference energy is taken as that of the separated $\mathrm{PH}_{3}$ and $\mathrm{He}$ species. In terms of anisotropy, the interaction between helium and phosphine exhibits a relatively strong anisotropy.

Fig. 2 shows that the 3D-PES has a unique potential well of $34.92 \mathrm{~cm}^{-1}$, which occurs at $\theta=90^{\circ}, \Phi=60^{\circ}$ and $R=6.75 \mathrm{bohr}$. This minimum is found at the same coordinates in Fig. 2(a) and (b), where the $2 \mathrm{D}$ cuts are periodical with a period of $120^{\circ}$ relative to the $\Phi$-axis, as expected for this $C_{3 v}$ symmetry molecule. Minimal energy configurations thus correspond to an $\mathrm{He}$ atom located between two $\mathrm{H}$ atoms and in the plane parallel to that formed by the three $\mathrm{H}$ atoms, whereas maxima of the potential occur where the $\mathrm{He}$ is in the plane containing the $\mathrm{P}, \mathrm{H}$ bond and $\mathrm{C}_{3}$ axis. 


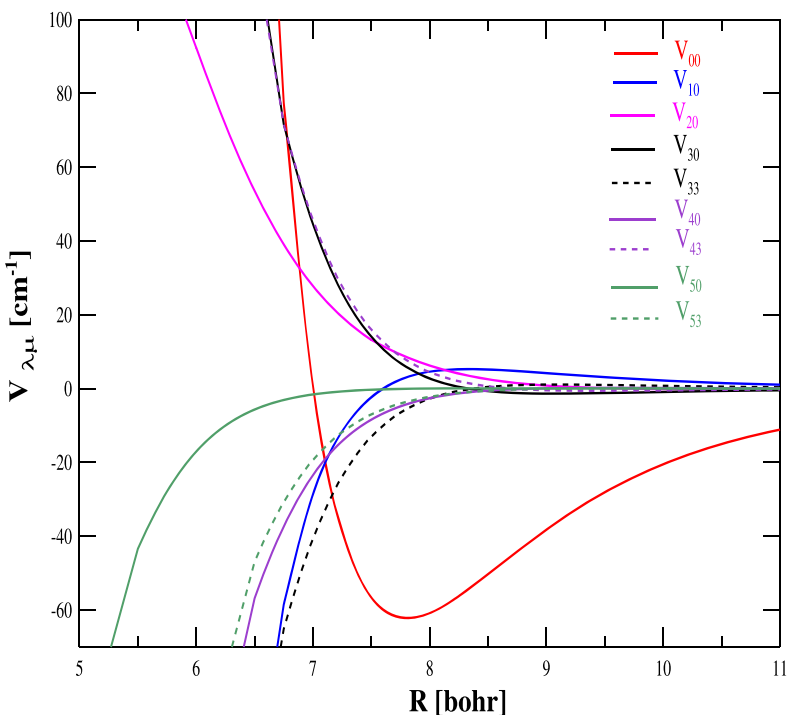

Figure 3. Dependence of selected radial coefficients (in units of $\mathrm{cm}^{-1}$ ) with respect to $R$.

\subsection{Analytic fit}

In order to derive the radial coefficients $V_{\lambda \mu}(R)$ required for the dynamical calculations, we expanded the 3D-PES of $\mathrm{PH}_{3}-\mathrm{He}$ over the normalized spherical harmonic functions $Y_{\lambda \mu}(\theta, \Phi)$ as follows:

$V(R, \theta, \Phi)=\sum_{\lambda \mu} V_{\lambda \mu}(R) Y_{\lambda \mu}(\theta, \Phi)$.

Due to the $C_{3 v}$ symmetry of the phosphine molecule, only radial terms for which $\mu=3 n$ ( $n$ being integer) are involved in the expansion (equation 2); the others are vanishing. Therefore, using the cubic spline routine, 70 radial coefficients $(0 \leq \lambda \leq 18$ and $0 \leq \mu \leq 18)$ were needed to reproduce the $a b$ initio potential reasonably within relative errors less than 5 per cent.

Fig. 3 depicts the variation of some radial coefficients $\left(V_{00}, V_{10}\right.$, $V_{20}, V_{30}, V_{33}, V_{40}$ and $V_{43}$ ) along the $R$ Jacobi coordinate. The $V_{00}$, $V_{20}, V_{30}$ and $V_{43}$ terms have upward concavities, while the others are oriented downward. A close examination of this figure reveals that, for $\mu=0$, the term related to $\lambda=3$ outweighs the others with respect to the isotropic radial coefficient, namely $V_{00}$. This is also the case for $\mu=3$ and $\lambda=4$. Therefore, no clear predominance pattern can be associated with the index pair $\lambda \mu$. This lack of well-defined propensity scheme will have consequences on the behaviour of crosssections. The radial coefficients derived from the $\mathrm{NH}_{3}-\mathrm{He}$ interacting potential show similar patterns, except for the terms involving $\mu=$ 3 (Gubbels et al. 2012). Typically, the terms $V_{33}$ and $V_{43}$ are oriented downward and upward (upward and downward) for $\mathrm{PH}_{3}-\mathrm{He}\left(\mathrm{NH}_{3}-\right.$ $\mathrm{He}$ ), respectively (i.e. they even present differences in sign at short range, as can be seen by comparison with fig. 3 of Gubbels et al. 2012).

\section{DYNAMICAL CALCULATIONS}

In this work, we are interested in the study of the rotational energy transfer of $\mathrm{PH}_{3}$ after collision with $\mathrm{He}$. $\mathrm{PH}_{3}$ is a symmetric top rotor type molecule. Usually, $j_{k}^{\epsilon}$ is used to label the energy levels of such molecules, where the quantum number $j$ stands for the total angular momentum and $k$ its projection over the $C_{3}$ axis, while $\epsilon= \pm$ denotes the symmetry index. For a given rotational energy level, the parity of the rotation-inversion wavefunction and

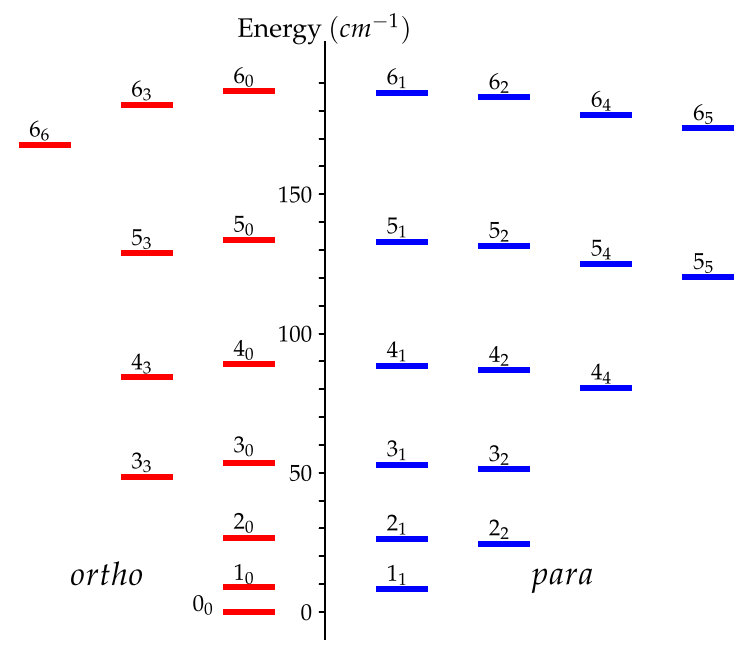

Figure 4. Diagram of the $\mathrm{PH}_{3}$ rotational energy levels $\left(E_{j k}\right)$. The energies are labelled as $j_{k}$. See text for the definition of these two quantum numbers. Their energies are given in Tables S1 and S2 in the Supplementary Material.

Table 1. Parameters used in the dynamical calculations to solve the coupled equations by means of the log derivative propagator. DTOL and OTOL stand for diagonal tolerance and off-diagonal tolerance, respectively.

\begin{tabular}{lc}
\hline ortho: $j_{k}^{\epsilon}=16_{16}^{0}$ & para: $j_{k}^{\epsilon}=14_{1}^{0}$ \\
$R_{\min }=3 a_{0}$ & $R_{\max }=35 a_{0}$ \\
STEPS $=10-200$ & $\mu=3.581 \mathrm{au}$ \\
DTOL $=0.01 \AA^{2}$ & OTOL $=0.005 \AA^{2}$ \\
\hline
\end{tabular}

the symmetry of the umbrella inversion are $(-1)^{j+k+1} \epsilon$ and $(-1)^{j+1} \epsilon$, respectively (Rist, Alexander \& Valiron 1993). Due to the $C_{3 v}$ symmetry of the $\mathrm{PH}_{3}$ molecule, the three hydrogen atoms of phosphine are identical in terms of spatial configuration. Thus, the rotational levels are split into ortho- $\mathrm{PH}_{3}\left(\mathrm{o}-\mathrm{PH}_{3}\right)$ and para- $\mathrm{PH}_{3}\left(\mathrm{p}-\mathrm{PH}_{3}\right)$, which stand for $k=3 n$ and $k \neq 3 n$ ( $n$ being integer), respectively. As $k$ can take any value except multiples of 3 , the $\mathrm{p}-\mathrm{PH}_{3}$ rotational energy levels are denser than the o- $\mathrm{PH}_{3}$ ones (see Fig. 4). These energy levels can be calculated using the spectroscopic constants $A=B=4.4524 \mathrm{~cm}^{-1}$ and $C=3.919 \mathrm{~cm}^{-1}$ by means of equation (3), where $\hbar^{2} / 2 I_{x}=X$ (Stroup, Oetjen \& Bell 1953):

$E_{j k}=\frac{\hbar^{2}}{2 I_{b}} j(j+1)+\left(\frac{\hbar^{2}}{2 I_{c}}-\frac{\hbar^{2}}{2 I_{b}}\right) k^{2}$.

As the conversion o- $\mathrm{PH}_{3}$ to $\mathrm{p}-\mathrm{PH}_{3}$ (and vice versa) is forbidden, the ortho and para scattering channels are treated separately in the cross-section computations.

\subsection{Cross-sections}

The quantum nuclear treatment detailed in this section was performed with the MOLSCAT computer code (Hutson \& Green 1994) using the time-independent close coupling method (Arthurs \& Dalgarno 1960), along with the log derivative propagator (Manolopoulos 1986). Preliminary tests were done in order to fix the parameters of the propagator (see Table 1), such as the integration boundaries ( $R_{\min }$ and $\left.R_{\max }\right)$, the size of the rotational basis $\left(j_{\max }\right.$ and $\left.k_{\max }\right)$ and the integration step, which depends on the variable STEPS. These optimized values have been selected within a convergence threshold of 1.0 percent $\AA^{2}$. Thereafter, the total energy $(E)$ was smoothly 
(a)

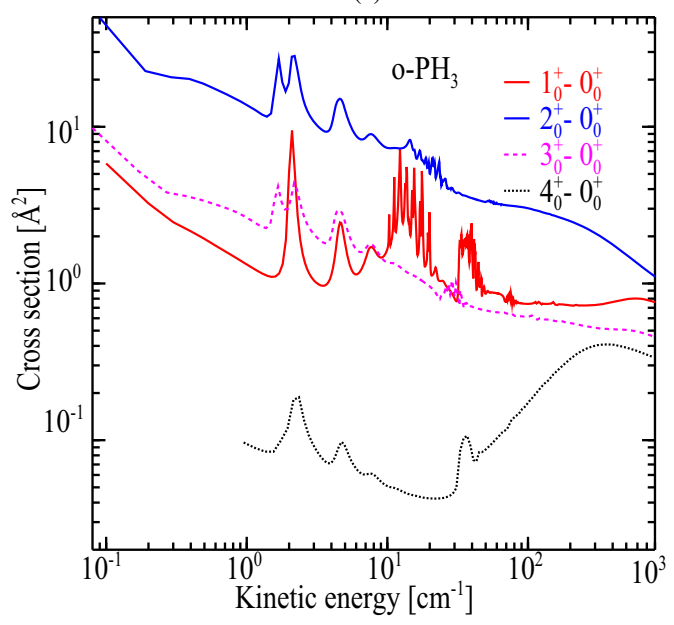

(b)

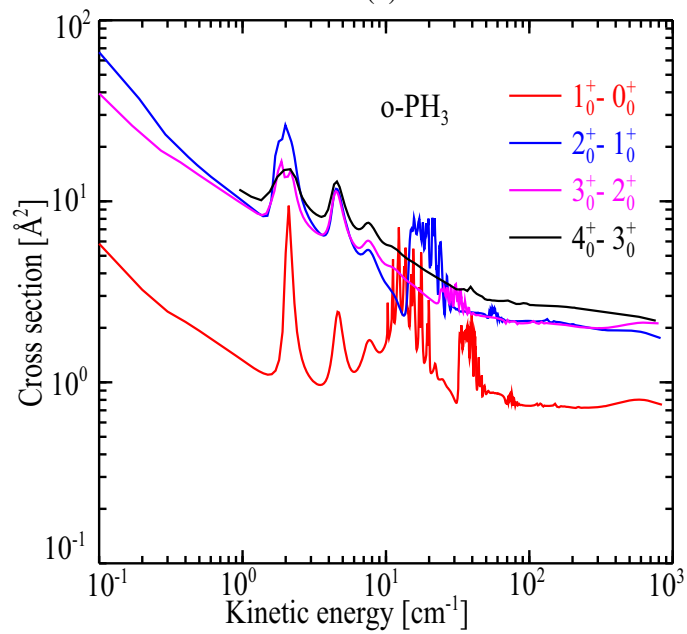

(c)

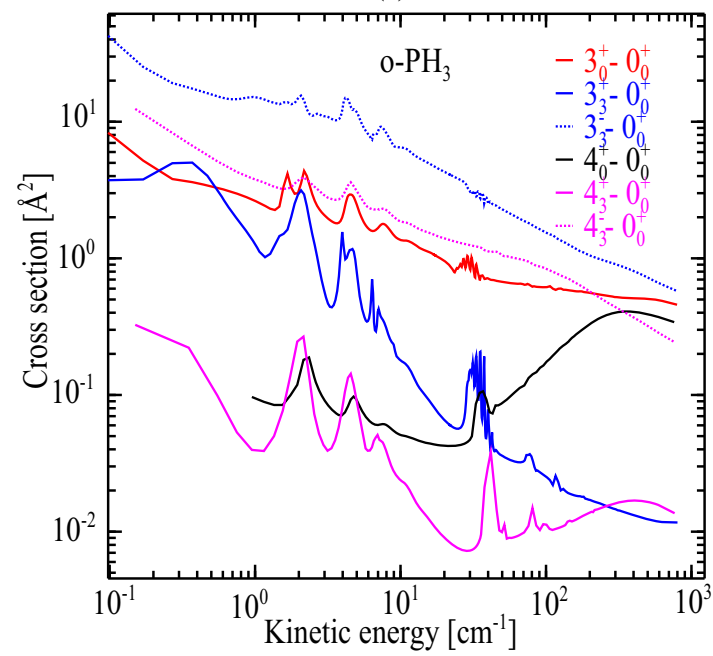

Figure 5. Inelastic cross-sections of o- $\mathrm{PH}_{3}$, induced by collision with He, versus the kinetic energy; panels (a), (b) and (c) are for de-excitations involving selected low-lying rotational levels.

varied from $9-1000 \mathrm{~cm}^{-1}\left(8.4-500 \mathrm{~cm}^{-1}\right)$ for the scattering of o$\mathrm{PH}_{3}\left(\mathrm{p}-\mathrm{PH}_{3}\right)$. In order to converge cross-sections properly, we take all open channels into account by adding some closed channels for both ortho and para. The convergence tests showed that a rotational basis set including 97 levels for $\mathrm{o}-\mathrm{PH}_{3}$ is large enough. The 97 th level corresponds to $j_{\max }=16$. For $\mathrm{p}-\mathrm{PH}_{3}$, we considered a basis set of 152 levels, corresponding to $j_{\max }=14$.

Fig. 5 displays the variation of cross-sections for $\mathrm{o}-\mathrm{PH}_{3}$ with respect to the kinetic energy. Fig. 5(a) depicts the $j_{0}^{+} \longrightarrow 0_{0}^{+}$ transitions, Fig. 5(b) represents the transitions for which $\{\Delta j, k$, $\epsilon\}=\{1,0,+\}$ and Fig. 5(c) shows the $j_{k}^{\epsilon} \longrightarrow 0_{0}^{+}$transitions. All these curves, except those associated with the $4_{0}^{+} \longrightarrow 0_{0}^{+}$and $4_{1}^{-} \longrightarrow 1_{1}^{+}$transitions, are decreasing while increasing the kinetic energy. Below $100 \mathrm{~cm}^{-1}$, these cross-sections present both Feshbach and shape resonances. Such features were expected, as they were found in previous works, such as the one treating the collisional excitation of $\mathrm{NH}_{3}$ by atomic and molecular hydrogen (Bouhafs et al. 2017) and that investigating the collisions between $\mathrm{NH}_{3}$ and He (Machin \& Roueff 2005). In fact, quasi-bound states occur when the He projectile is caught in the potential well, leading to Feshbach resonances. Regarding the shape resonances, they manifest when tunnelling effects via the centrifugal energy barrier take place, forming quasi-bound states.

\subsection{1 o- $\mathrm{PH}_{3}$}

Fig. 5(a) shows that the cross-sections of the $2_{0}^{+} \longrightarrow 0_{0}^{+}$and $4_{0}^{+} \longrightarrow$ $0_{0}^{+}$transitions are the strongest and weakest ones, respectively, in terms of magnitude, while an inversion occurs between the $1_{0}^{+} \longrightarrow$ $0_{0}^{+}$and $3_{0}^{+} \longrightarrow 0_{0}^{+}$transition cross-sections. Therefore, no selection rules can be associated with the parity of $j$. From Fig. 5(a) and (b), one can see that the magnitude of resonances decreases when $j$ increases. Fig. 5(c) shows that the cross-sections of the $3_{3}^{+} \longrightarrow 0_{0}^{+}\left(4_{3}^{+} \longrightarrow 0_{0}^{+}\right)$ and $3_{3}^{-} \longrightarrow 0_{0}^{+}\left(4_{3}^{-} \longrightarrow 0_{0}^{+}\right)$transitions are from either side of the $3_{0}^{+} \longrightarrow 0_{0}^{+}\left(4_{0}^{+} \longrightarrow 0_{0}^{+}\right)$transition. Referring to each $j_{0}^{+} \longrightarrow 0_{0}^{+}$, the cross-sections of the $j_{3}^{-} \longrightarrow 0_{0}^{+}$transitions are lifted, while those of the $j_{3}^{+} \longrightarrow 0_{0}^{+}$ones are shifted downward. Therefore, the propensity rule is in favour of symmetry (or parity) breaking, i.e. $\Delta k=3$ and $j \longrightarrow 0$. In addition, each cross-section for the $j_{0}^{+} \longrightarrow 0_{0}^{+}$transition takes the shape of that of the $j_{3}^{-} \longrightarrow 0_{0}^{+}$transition at low kinetic energy and then switches progressively to that of $j_{3}^{+} \longrightarrow 0_{0}^{+}$. 
(d)

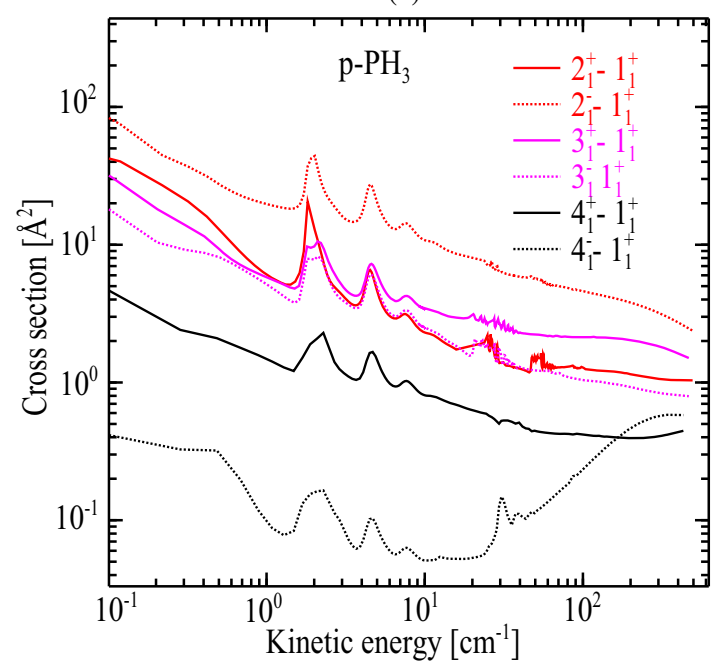

(e)

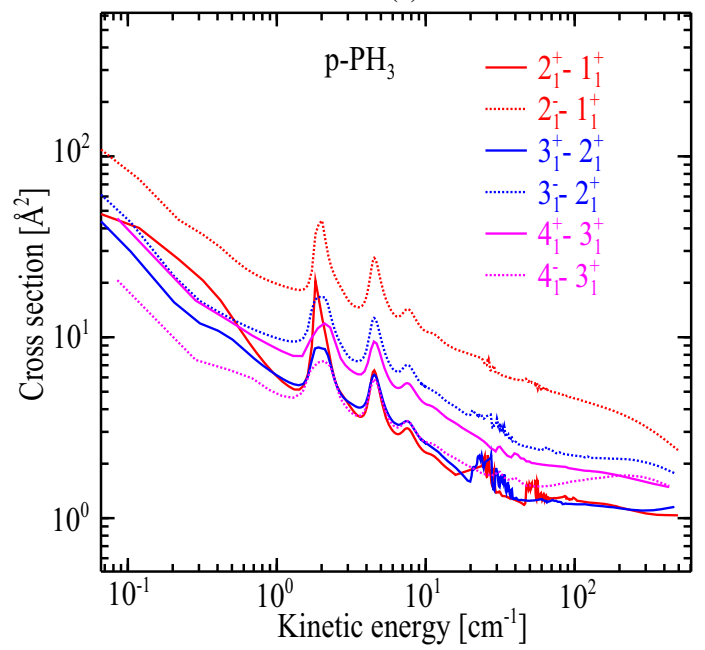

(f)

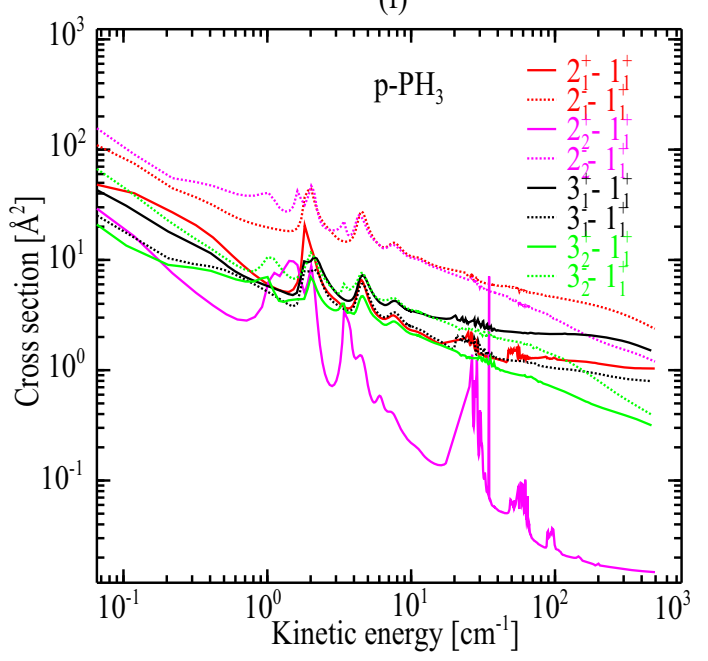

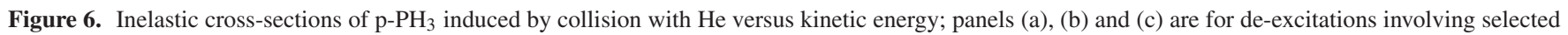
low-lying rotational levels.

\subsection{2 $\mathrm{p}-\mathrm{PH}_{3}$}

Fig. 6 displays the variation of cross-sections for $\mathrm{p}-\mathrm{PH}_{3}$ with respect to the kinetic energy. Fig. 6(a) shows that the magnitude of crosssections decreases when $j$ increases for the $j_{1}^{-} \longrightarrow 1_{1}^{+}$transitions, while for the $j_{1}^{+} \longrightarrow 1_{1}^{+}$transitions no net dominance is observed. In Fig. 6(b), all cross-sections have almost the same shape, whatever the transition, with typically the same location of resonance peaks. Regarding the magnitudes, the transitions involving negative parities are stronger than the corresponding ones with positive parities, except for $j=4$. For instance, the cross-section of the $2_{1}^{-} \longrightarrow 1_{1}^{+}$ $\left(4_{1}^{+} \longrightarrow 3_{1}^{+}\right)$transition outweighs that of $2_{1}^{+} \longrightarrow 1_{1}^{+}\left(4_{1}^{-} \longrightarrow 3_{1}^{+}\right)$. Thus, one can state the predominance of parity breaking; $\Delta k=0$ and $\Delta j=1$ is lifted for higher $j$ values. Fig. 6(c) is an extension of Fig. 6 (b) to show the effect of the change of $\Delta k$. The previously highlighted behaviour persists, but for fixed $j$ and $\epsilon$. The slight dominance of $k=2$ occurs only at low kinetic energy, then it reverses at higher energies (e.g. the $2_{1}^{-} \longrightarrow 1_{1}^{+}$and $2_{2}^{-} \longrightarrow 1_{1}^{+}$ transitions).
In summary, we can conclude that no general propensity rule can be associated with the indexes $j, k, \epsilon$, in line with the already noticed behaviours of the radial coefficients (see Fig. 3).

\subsection{Rate coefficients}

In order to deduce the rotational rate coefficients $\left(R_{j k \epsilon \rightarrow j^{\prime} k^{\prime} \epsilon^{\prime}}\right)$ of both o- $\mathrm{PH}_{3}$ and p- $\mathrm{PH}_{3}$, we averaged the cross-sections discussed above thermally using the Maxwell-Boltzmann velocity distribution:

$$
R_{\alpha \rightarrow \alpha^{\prime}}(T)=\left(\frac{8}{\pi \mu \beta}\right)^{1 / 2} \beta^{2} \int_{0}^{\infty} E_{c} \sigma_{\alpha \rightarrow \alpha^{\prime}}\left(E_{c}\right) e^{-\beta E_{c}} \mathrm{~d} E_{c},
$$

where the kinetic energy is denoted by $E_{c}, \beta$ stands for the reverse of $\left(k_{\mathrm{B}} \times T\right)$, with $k_{\mathrm{B}}$ being the constant of Boltzmann, and $\alpha$ refers to $j k \epsilon$.

The cross-sections of ortho- $\mathrm{PH}_{3}$ covering the range $9-1000 \mathrm{~cm}^{-1}$ allow us to generate the velocity coefficient for the transitions 

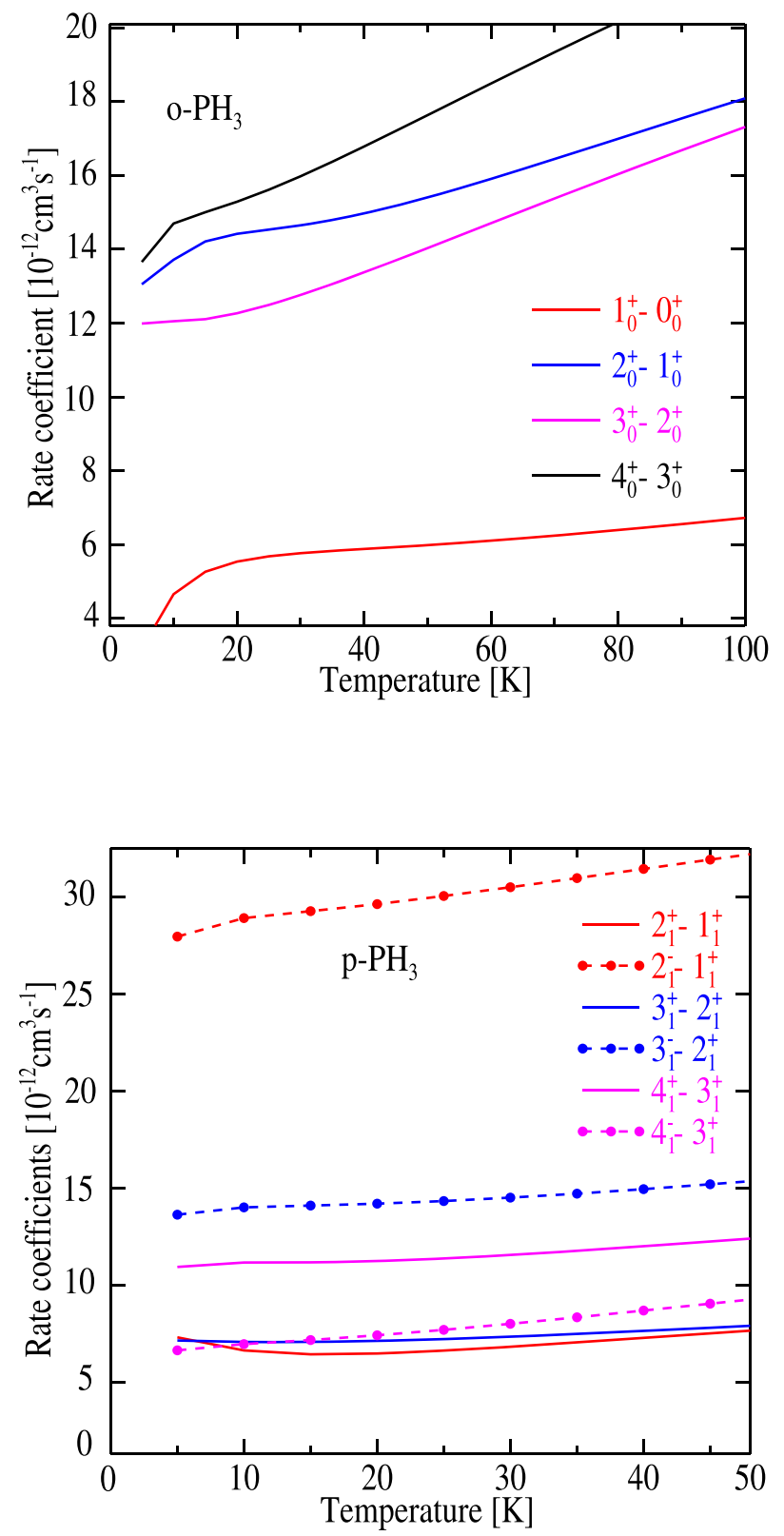

Figure 7. Downward rotational rate coefficients of o- $\mathrm{PH}_{3}$ (top panel) and p$\mathrm{PH}_{3}$ (bottom panel) induced by collision with $\mathrm{He}$, as a function of the kinetic temperature.

between the 41 low-lying rotational levels [up to $j_{k}=10_{0}$ ] for temperatures up to $100 \mathrm{~K}$. Similary, the cross-sections of para- $\mathrm{PH}_{3}$ covering the range $8.4-500 \mathrm{~cm}^{-1}$ allow us to generate the velocity coefficient for the transitions between the 41 low-lying rotational levels [up to $j_{k}=7_{1}$ ] for temperatures up to $50 \mathrm{~K}$.

Fig. 7 displays the variation of rate coefficients for the rotational excitation of o- $\mathrm{PH}_{3}$ and $\mathrm{p}-\mathrm{PH}_{3}$ due to collisions with $\mathrm{He}$ as a function of the kinetic temperature. We represented only a few curves, as the patterns observed for the cross-sections persist here, whereas the full set of data is given in the Supplementary Material. Typically, for o- $\mathrm{PH}_{3}$, the top panel of Fig. 7 reproduces the behaviour observed in Fig. 5(b). The main difference consists of the dominance of the $4_{0}^{+} \longrightarrow 3_{0}^{+}$transition, which occurs for the whole temperature range, and no inversion is observed among the $2_{0}^{+} \longrightarrow 1_{0}^{+}$and $3_{0}^{+} \longrightarrow 2_{0}^{+}$ transitions. Regarding p- $\mathrm{PH}_{3}$, the bottom panel of Fig. 7 confirms
Table 2. Comparison of $\mathrm{PH}_{3}$ (this work) and $\mathrm{NH}_{3}$ (Machin \& Roueff 2005) rotational rate coefficients (in units of $\mathrm{cm}^{3} \mathrm{~s}^{-1}$ ) at selected temperatures. The brackets stand for negative powers of ten.

\begin{tabular}{|c|c|c|c|c|c|c|c|c|c|}
\hline \multicolumn{3}{|c|}{ Initial levels } & \multicolumn{3}{|c|}{ Final levels } & \multicolumn{2}{|c|}{$T=50 \mathrm{~K}$} & \multicolumn{2}{|c|}{$T=100 \mathrm{~K}$} \\
\hline$j$ & $k$ & $\epsilon$ & $j^{\prime}$ & $k^{\prime}$ & $\epsilon^{\prime}$ & o- $\mathrm{PH}_{3}$ & $\mathrm{o}-\mathrm{NH}_{3}$ & o- $\mathrm{PH}_{3}$ & $\mathrm{o}-\mathrm{NH}_{3}$ \\
\hline 1 & 0 & + & 0 & 0 & + & $5.99(12)$ & $2.72(12)$ & $6.73(12)$ & $4.65(12$ \\
\hline 2 & 0 & + & 0 & 0 & + & $2.09(11)$ & $1.37(11)$ & $2.34(11)$ & $1.72(11)$ \\
\hline 2 & 0 & + & 1 & 0 & + & $1.54(11)$ & $0.59(11)$ & $1.81(11)$ & $0.88(11)$ \\
\hline 3 & 3 & - & 0 & 0 & + & $1.43(11)$ & $1.19(11)$ & $1.34(11)$ & $1.45(11)$ \\
\hline 3 & 3 & - & 2 & 0 & + & $3.20(11)$ & $1.61(11)$ & $3.21(11)$ & $2.36(11)$ \\
\hline 3 & 3 & + & 0 & 0 & + & $3.70(13)$ & $0.11(13)$ & $2.80(13)$ & $0.14(13$ \\
\hline 3 & 3 & + & 2 & 0 & + & $2.24(11)$ & $0.23(11)$ & $2.15(11)$ & $0.45(11)$ \\
\hline 4 & 0 & + & 3 & 0 & + & $1.76(11)$ & $0.66(11)$ & $2.17(11)$ & $0.98(11$ \\
\hline 4 & 0 & + & 0 & 0 & + & $6.82(13)$ & $1.76(13)$ & $1.66(12)$ & $3.61(13$ \\
\hline \multirow[t]{2}{*}{4} & 3 & + & 0 & 0 & + & $7.40(14)$ & $1.51(14)$ & $1.00(13)$ & $0.25(13$ \\
\hline & & & & & & \multicolumn{2}{|c|}{$T=25 \mathrm{~K}$} & \multicolumn{2}{|c|}{$T=50 \mathrm{~K}$} \\
\hline$j$ & $k$ & $\epsilon$ & $j^{\prime}$ & $k^{\prime}$ & $\epsilon^{\prime}$ & $\mathrm{p}-\mathrm{PH}_{3}$ & $\mathrm{p}-\mathrm{NH}_{3}$ & p- $-\mathrm{PH}_{3}$ & $\mathrm{p}-\mathrm{NH}_{3}$ \\
\hline 2 & 2 & + & 1 & 1 & + & 2.61(13) & $0.81(13)$ & $2.23(13)$ & $0.54(13$ \\
\hline 2 & 2 & - & 1 & 1 & + & $2.70(11)$ & $1.39(11)$ & $2.57(11)$ & $1.70(11)$ \\
\hline 2 & 1 & + & 1 & 1 & + & $6.63(12)$ & $3.10(12)$ & $7.76(12)$ & $3.98(12$ \\
\hline 2 & 1 & - & 1 & 1 & + & $3.01(11)$ & $3.20(11)$ & $3.24(11)$ & $3.47(11)$ \\
\hline 3 & 2 & + & 1 & 1 & + & $5.60(12)$ & $1.98(12)$ & $5.58(12)$ & $2.60(12$ \\
\hline 3 & 2 & - & 1 & 1 & + & $9.75(12)$ & $0.19(12)$ & $1.02(11)$ & $0.14(11$ \\
\hline 3 & 1 & + & 2 & 1 & + & $7.23(12)$ & $3.56(12)$ & $7.98(12)$ & 4.61(12) \\
\hline 3 & 1 & - & 2 & 1 & + & $1.43(11)$ & $1.08(11)$ & $1.55(11)$ & $1.26(11)$ \\
\hline 4 & 1 & + & 3 & 1 & + & $1.14(11)$ & $0.41(11)$ & $1.25(11)$ & $0.54(11)$ \\
\hline 4 & 1 & - & 3 & 1 & + & 7.71(12) & $3.77(12)$ & $9.41(12)$ & $0.52(12$ \\
\hline
\end{tabular}

the dominance in favour of the de-excitations for which the parity is broken, $\Delta k=0$ and $j \leq 3 \longrightarrow j^{\prime}$, as can be seen in panel $(e)$ of Fig. 5.

It is worth noting that the rate coefficients of $\mathrm{NH}_{3}$ induced by collisions with helium (Machin \& Roueff 2005), supplemented by those due to para- $\mathrm{H}_{2}$ impact (Rist et al. 1993), were used to model the $\mathrm{PH}_{3}$ abundance in astrophysical media. In this realm, comparing our data on $\mathrm{PH}_{3}-\mathrm{He}$ with those of Machin \& Roueff (2005) on $\mathrm{NH}_{3}-\mathrm{He}$ is a clue to settle the validity of using rate coefficients of isoelectronic molecules instead. For this purpose, Table 2 compares the $\mathrm{PH}_{3}$ rate coefficients computed in this work with those of $\mathrm{NH}_{3}$ (Machin \& Roueff 2005). This table shows that large differences between both sets of data are observed for both ortho and para, especially for $j \geq$ 3. For $\Delta j=1$ (as in the observed transitions, $1_{0}^{+} \longrightarrow 0_{0}^{+}, 2_{0}^{+} \longrightarrow 1_{0}^{+}$ and $2_{1}^{\epsilon} \longrightarrow 1_{1}^{\epsilon}$ ), we noticed agreement within a factor of $\sim 2.5$. In order to have a global view of the comparison, in Fig. 8 we plot the rate coefficients of $\mathrm{PH}_{3}$ as a function of those of $\mathrm{NH}_{3}$. For both ortho and para, the data reported by Machin \& Roueff (2005) are smaller than the values computed in this work. At low temperature, the rate coefficients of $\mathrm{NH}_{3}$ can drop down to a factor of two orders of magnitude lower than those of $\mathrm{PH}_{3}$. This discrepancy decreases as the temperature increases, which suggests a better agreement at higher temperatures $(T \geq 300)$. Although $\mathrm{PH}_{3}$ is observed in a relatively hot environment (e.g. the carbon star envelopes IRC+10216 and CRL2688), using the rate coefficients of $\mathrm{NH}_{3}$ to model its abundance will lead to an underestimation of its density. Consequently, this may lead to misunderstandings in phosphorous chemistry, as phosphine is likely one of the main phosphorous reservoirs in space. Otherwise, the observed lines suggest that phosphine can be formed anywhere in a disc of radius $100 R_{\star}$ around the centre of the star (Agúndez et al. 2014). Hence, $\mathrm{PH}_{3}$ can eventually be observed in warm/cold media, where the use of $\mathrm{NH}_{3}$ rate coefficients would yield underestimation of the abundance of phosphine by a factor larger than one order of magnitude. Consequently, the present data should be used, rather than those of $\mathrm{NH}_{3}$, for more reliable determination of the $\mathrm{PH}_{3}$ abundances in space. 

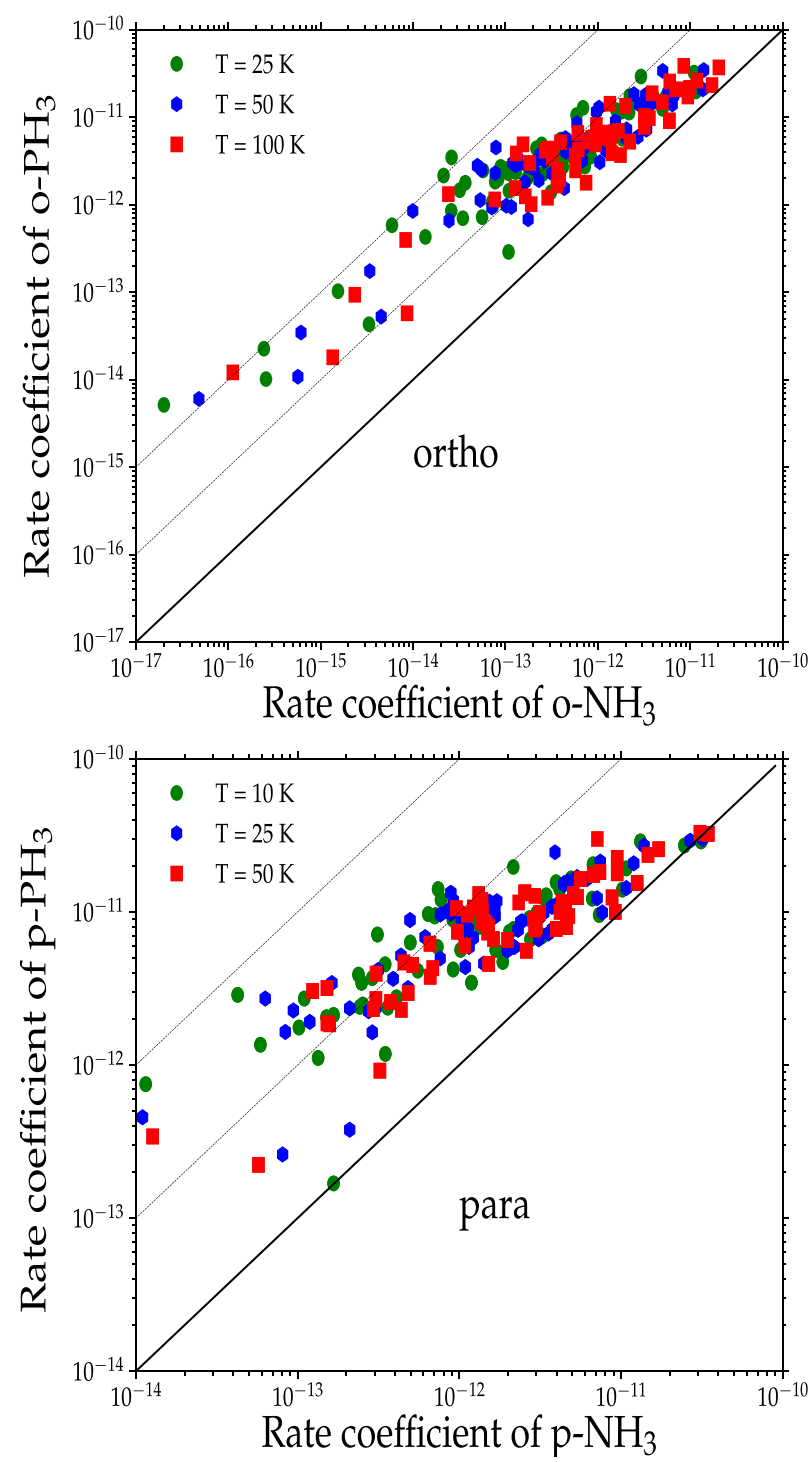

Figure 8. Comparison of the $\mathrm{PH}_{3}$ rate coefficients $\left(\right.$ in $\mathrm{cm}^{3} \mathrm{~s}^{-1}$ ) with those of $\mathrm{NH}_{3}$. With respect to the diagonal, the first dashed line represents a factor of one order of magnitude and the second dashed line stands for a factor of two orders of magnitude.

\section{CONCLUSION}

We carried out the construction of a highly accurate 3D-PES for the $\mathrm{PH}_{3}-\mathrm{He}$ interacting system. This 3D-PES was mapped in Jacobi coordinates and computed at the $\operatorname{CCSD}(\mathrm{T}) /$ aug-cc-pVQZ+bf level of theory. The interaction among helium and phosphine is quite anisotropic and presents one single potential well of $34.92 \mathrm{~cm}^{-1}$ at $R=6.75$ bohr, $\theta=90^{\circ}$ for $\Phi=60^{\circ}$ and $\Phi=180^{\circ}$. Then, an analytical expansion of this 3D-PES was derived. These electronic structure computations were followed by computations of state-tostate inelastic cross-sections of rotational (de-)excitation of orthoand para-phosphine, induced by collision with helium. This is done using a quantum mechanical treatment, by means of the close-coupling approach. These data were then thermally averaged according to the Maxwell-Boltzmann kinetic energy distribution, leading to record rate coefficients up to (100 K) $50 \mathrm{~K}$ for the (41) 42 low-lying rotational levels of $\left(\mathrm{o}-\mathrm{PH}_{3}\right) \mathrm{p}-\mathrm{PH}_{3}$. No general propensity rules are found for either $\mathrm{p}-\mathrm{PH}_{3}$ or $\mathrm{o}-\mathrm{PH}_{3}$.
The rate coefficients computed in this work were compared with those of Machin \& Roueff (2005) for the $\mathrm{NH}_{3}-\mathrm{He}$ system, which is used to estimate the abundances of phosphine. Large differences are noticed, especially at low temperatures. Therefore, the results obtained in this work may be of great help to the astrophysical community, in order to model the physical conditions of media where phosphine was observed. Indeed, exact knowledge of the $\mathrm{PH}_{3}$ abundance may be a clue to constrain the chemistry of interstellar phosphorus. In addition, the present data may help in the identification of $\mathrm{PH}_{3}$ in astrophysical media where other P-containing molecules were detected, e.g. in massive dense cores (Fontani et al. 2016) or the Galactic Centre (Rivilla et al. 2018).

\section{ACKNOWLEDGEMENTS}

The authors acknowledge the Programme National 'Physique et Chimie du Milieu Interstellaire' (PCMI) of Centre National de la Recherche Scientifique (CNRS).

\section{DATA AVAILABILITY}

Collision rates are available in the Supplementary Material. The strongest inelastic transitions are of the order of $10^{-11}$; we considered as significant only values of rates that are greater than $1 \times 10^{-14}$. Values that are less than $1 \times 10^{-14}$ are considered significantly zero.

\section{REFERENCES}

Agúndez M., Cernicharo J., Guélin M., 2007, ApJ, 662, L91

Agúndez M., Cernicharo J., Pardo J., Guélin M., Phillips T., 2008, A\&A, 485, L33

Agúndez M., Cernicharo J., Decin L., Encrenaz P., Teyssier D., 2014, ApJ, 790, L27

Altwegg K. B. H. B.-N.-A. E. A. O., 2016, Science Advances, 2, e1600285

Arnett D., Arnett W. D., 1996, Supernovae and Nucleosynthesis: An Investigation of the History of Matter, From the Big Bang to the Present. Princeton University Press

Arthurs A., Dalgarno A., 1960, 540

Barlow M. et al., 2013, Science, 342, 1343

Bop C. T., Hammami K., Niane A., Faye N., Jaïdane N., 2016, MNRAS, p. stw2809

Bouhafs N., Rist C., Daniel F., Dumouchel F., Lique F., Wiesenfeld L., Faure A., 2017, MNRAS, 470, 2204

Boys S. F., Bernardi F. D., 1970, Molecular Physics, 19, 553

Bregman J. D. L. D. F., Rank D. M., 1975, ApJ, 202, L55

Cernicharo J. et al., 2018, ApJ, 853, L22

Chantzos J., Rivilla V. M., Vasyunin A., Redaelli E., Bizzocchi L., Fontani F., Caselli P., 2020, A\&A, 633, A54

Cybulski S. M., Toczyłowski R. R., 1999, The Journal of Chemical Physics, 111,10520

Danby G., Flower D., Valiron P., Schilke P., Walmsley C., 1988, MNRAS, 235,229

De Beck E., Kamiński T., Patel N., Young K., Gottlieb C., Menten K., Decin L., 2013, A\&A, 558, A132

Dunning T. H., Jr, 1989, The Journal of Chemical Physics, 90, 1007

El Hanini H., Najar F., Naouai M., Jaidane N.-E., 2019, Physical Chemistry Chemical Physics

Faure A., Valiron P., Wernli M., Wiesenfeld L., Rist C., Noga J., Tennyson J., 2005, A full nine-dimensional potential-energy surface for hydrogen molecule-water collisions

Fontani F., Rivilla V., Caselli P., Vasyunin A., Palau A., 2016, ApJ, 822, L30

Gubbels K. B., Meerakker S. Y. V. D., Groenenboom G. C., Meijer G., Avoird A. V. D., 2012, The Journal of Chemical Physics, 136, 074301

Guélin M., Cernicharo J., Paubert G., Turner B., 1990, A\&A, 230, L9

Halfen D., Clouthier D., Ziurys L. M., 2008, ApJ, 677, L101 
Herzberg G., 1966, Van Nostrand, Reinhold, New York

Hutson J., Green S., 1994, Collaborative computational project

Jiménez-Serra I., Viti S., Quénard D., Holdship J., 2018, ApJ, 862, 128

Larson H., Fink U., Smith H., Davis D., 1980, ApJ, 240, 327

Larson H. P. F. U. T.-R. R., 1977, ApJ, 211, 972

Levy A., Lacome N., Tarrago G., 1994, Journal of Molecular Spectroscopy, 166, 20

Machin L., Roueff E., 2005, Journal of Physics B: Atomic, Molecular and Optical Physics, 38, 1519

Maciá E., 2005, Chemical Society Reviews, 34, 691

Manolopoulos D., 1986, The Journal of Chemical Physics, 85, 6425

Milam S., Halfen D., Tenenbaum E., Apponi A., Woolf N., Ziurys L., 2008, ApJ, 684, 618

Moreno R., Marten A., Lellouch E., 2009, in AAS/Division for Planetary Sciences Meeting Abstracts\# 41

Najar F., Naouai M., Hanini H. E., Jaidane N., 2017, MNRAS, 472, 2919

Pickett H., Poynter R., Cohen E., 1981, Journal of Quantitative Spectroscopy and Radiative Transfer, 26, 197

Ridgway S., Wallace L., Smith G., 1976, ApJ, 207, 1002

Rist C., Alexander M. H., Valiron P., 1993, The Journal of Chemical Physics, 98,4662

Rivilla V. et al., 2018, MNRAS, 475, L30

Salem J., Bouanich J.-P., Walrand J., Aroui H., Blanquet G., 2005, Journal of Molecular Spectroscopy, 232, 247

Sharp C. M., Burrows A., 2007, ApJS, 168, 140

Sousa-Silva C., Al-Refaie A. F., Tennyson J., Yurchenko S. N., 2015, MNRAS, 446, 2337

Stroup R. E., Oetjen R. A., Bell E. E., 1953, JOSA, 43, 1096

Tenenbaum E., Ziurys L. M., 2008a, ApJ, 680, L121

Tenenbaum E., Ziurys L. M., 2008b, ApJ, 680, L121

Tenenbaum E., Woolf N., Ziurys L. M., 2007, ApJ, 666, L29

Turner B., Bally J., 1987, ApJ, 321, L75

Turner B., Tsuji T., Bally J., Guelin M., Cernicharo J., 1990, ApJ, 365, 569

Watts J. D., Gauss J., Bartlett R. J., 1993, The Journal of Chemical Physics, 98,8718
Weisstein E. W., Serabyn E., 1996, Icarus, 123, 23

Werner H.-J. et al., 2010, see http://www.molpro.net

Woosley S., 2002, Rev. Mod. Phys., 74, 1015

Ziurys L. M., 1987, ApJ, 321, L81

\section{SUPPORTING INFORMATION}

Supplementary data are available at MNRAS online.

Table S1: Rotational levels of ortho- $\mathrm{PH}_{3}$.

Table S2: Rotational levels of para- $\mathrm{PH}_{3}$.

Table S3: Rate coefficients (in units of $\mathrm{cm}^{3} \mathrm{~s}^{-1}$, given as $A(B)=A$ $\times 10^{-B}$ ) of ortho- $\mathrm{PH}_{3}$ induced by collision with $\mathrm{He}$ as a function of the kinetic temperature, where (II), IF corresponds to (Initial), Final-Index).

Table S4: Rate coefficients (in units of $\mathrm{cm}^{3} \mathrm{~s}^{-1}$, given as $A(B)=A$ $\times 10^{-B}$ ) of ortho- $\mathrm{PH}_{3}$ induced by collision with $\mathrm{He}$ as a function of the kinetic temperature, where (II), IF corresponds to (Initial), Final-Index).

Table S5: Rate coefficients (in units of $\mathrm{cm}^{3} \mathrm{~s}^{-1}$, given as $A(B)=$ $A \times 10^{-B}$ ) of para- $\mathrm{PH}_{3}$ induced by collision with $\mathrm{He}$ as a function of the kinetic temperature, where (II), IF corresponds to (Initial), Final-Index).

Please note: Oxford University Press is not responsible for the content or functionality of any supporting materials supplied by the authors. Any queries (other than missing material) should be directed to the corresponding author for the article.

This paper has been typeset from a $\mathrm{T}_{\mathrm{E}} \mathrm{X} / \mathrm{L} \mathrm{T} \mathrm{E} \mathrm{X}$ file prepared by the author. 


\section{List of astronomical key words (Updated on 2020 January)}

This list is common to Monthly Notices of the Royal Astronomical Society, Astronomy and Astrophysics, and The Astrophysical Journal. In order to ease the search, the key words are subdivided into broad categories. No more than six subcategories altogether should be listed for a paper.

The subcategories in boldface containing the word 'individual' are intended for use with specific astronomical objects; these should never be used alone, but always in combination with the most common names for the astronomical objects in question. Note that each object counts as one subcategory within the allowed limit of six.

The parts of the key words in italics are for reference only and should be omitted when the keywords are entered on the manuscript.

\section{General}

editorials, notices

errata, addenda

extraterrestrial intelligence

history and philosophy of astronomy

miscellaneous

obituaries, biographies

publications, bibliography

sociology of astronomy

standards

\section{Physical data and processes}

acceleration of particles

accretion, accretion discs

asteroseismology

astrobiology

astrochemistry

astroparticle physics

atomic data

atomic processes

black hole physics

chaos

conduction

convection

dense matter

diffusion

dynamo

elementary particles

equation of state

gravitation

gravitational lensing: micro

gravitational lensing: strong

gravitational lensing: weak

gravitational waves

hydrodynamics

instabilities

line: formation

line: identification

line: profiles

magnetic fields

magnetic reconnection

(magnetohydrodynamics) MHD

masers

molecular data

molecular processes

neutrinos

nuclear reactions, nucleosynthesis, abundances

opacity

plasmas

polarization radiation: dynamics

radiation mechanisms:general

radiation mechanisms: non-thermal

radiation mechanisms: thermal

radiative transfer

relativistic processes

scattering

shock waves

solid state: refractory

solid state: volatile

turbulence

waves

\author{
Astronomical instrumentation, methods and techniques \\ atmospheric effects \\ balloons \\ instrumentation: adaptive optics \\ instrumentation: detectors \\ instrumentation: high angular resolution \\ instrumentation: interferometers \\ instrumentation: miscellaneous \\ instrumentation: photometers \\ instrumentation: polarimeters \\ instrumentation: spectrographs \\ light pollution \\ methods: analytical \\ methods: data analysis \\ methods: laboratory: atomic \\ methods: laboratory: molecular \\ methods: laboratory: solid state \\ methods: miscellaneous \\ methods: numerical \\ methods: observational \\ methods: statistical \\ site testing \\ space vehicles \\ space vehicles: instruments \\ techniques: high angular resolution \\ techniques: image processing \\ techniques: imaging spectroscopy \\ techniques: interferometric \\ techniques: miscellaneous \\ techniques: photometric \\ techniques: polarimetric \\ techniques: radar astronomy \\ techniques: radial velocities \\ techniques: spectroscopic \\ telescopes
}




\section{Astronomical data bases}

astronomical data bases: miscellaneous

atlases

catalogues

surveys

virtual observatory tools

\section{Software}

software: data analysis

software: development

software: documentation

software: public release

software: simulations

\author{
Astrometry and celestial mechanics \\ astrometry \\ celestial mechanics \\ eclipses \\ ephemerides \\ occultations \\ parallaxes \\ proper motions \\ reference systems \\ time
}

\section{The Sun}

Sun: abundances

Sun: activity

Sun: atmosphere

Sun: chromosphere

Sun: corona

Sun: coronal mass ejections (CMEs)

Sun: evolution

Sun: faculae, plages

Sun: filaments, prominences

Sun: flares

Sun: fundamental parameters

Sun: general

Sun: granulation

Sun: helioseismology

Sun: heliosphere

Sun: infrared

Sun: interior

Sun: magnetic fields

Sun: oscillations

Sun: particle emission

Sun: photosphere

Sun: radio radiation

Sun: rotation

(Sun:) solar-terrestrial relations

(Sun:) solar wind

(Sun:) sunspots

Sun: transition region

Sun: UV radiation

Sun: X-rays, gamma-rays

\section{Planetary systems}

comets: general

comets: individual: ... .

Earth

interplanetary medium

Kuiper belt: general

Kuiper belt objects: individual: . . . meteorites, meteors, meteoroids minor planets, asteroids: general

minor planets, asteroids: individual: ...

Moon

Oort Cloud

planets and satellites: atmospheres

planets and satellites: aurorae

planets and satellites: composition

planets and satellites: detection

planets and satellites: dynamical evolution and stability

planets and satellites: formation

planets and satellites: fundamental parameters

planets and satellites: gaseous planets

planets and satellites: general

planets and satellites: individual: ...

planets and satellites: interiors

planets and satellites: magnetic fields

planets and satellites: oceans

planets and satellites: physical evolution

planets and satellites: rings

planets and satellites: surfaces

planets and satellites: tectonics

planets and satellites: terrestrial planets

planet-disc interactions

planet-star interactions

protoplanetary discs

zodiacal dust

Stars

stars: abundances

stars: activity

stars: AGB and post-AGB

stars: atmospheres

(stars:) binaries (including multiple): close

(stars:) binaries: eclipsing

(stars:) binaries: general

(stars:) binaries: spectroscopic

(stars:) binaries: symbiotic

(stars:) binaries: visual

stars: black holes

(stars:) blue stragglers

(stars:) brown dwarfs

stars: carbon

stars: chemically peculiar

stars: chromospheres

(stars:) circumstellar matter

stars: coronae

stars: distances

stars: dwarf novae

stars: early-type

stars: emission-line, Be

stars: evolution

stars: flare

stars: formation

stars: fundamental parameters

(stars:) gamma-ray burst: general

(stars:) gamma-ray burst: individual: ...

stars: general

(stars:) Hertzsprung-Russell and colour-magnitude

diagrams

stars: horizontal branch

stars: imaging

stars: individual: ...

stars: interiors 
stars: jets

stars: kinematics and dynamics

stars: late-type

stars: low-mass

stars: luminosity function, mass function

stars: magnetars

stars: magnetic field

stars: massive

stars: mass-loss

stars: neutron

(stars:) novae, cataclysmic variables

stars: oscillations (including pulsations)

stars: peculiar (except chemically peculiar)

(stars:) planetary systems

stars: Population II

stars: Population III

stars: pre-main-sequence

stars: protostars

(stars:) pulsars: general

(stars:) pulsars: individual: ...

stars: rotation

stars: solar-type

(stars:) starspots

stars: statistics

(stars:) subdwarfs

(stars:) supergiants

(stars:) supernovae: general

(stars:) supernovae: individual: ...

stars: variables: Cepheids

stars: variables: Scuti

stars: variables: general

stars: variables: RR Lyrae

stars: variables: S Doradus

stars: variables: T Tauri, Herbig Ae/Be

(stars:) white dwarfs

stars: winds, outflows

stars: Wolf-Rayet

Interstellar medium (ISM), nebulae

ISM: abundances

ISM: atoms

ISM: bubbles

ISM: clouds

(ISM:) cosmic rays

(ISM:) dust, extinction

ISM: evolution

ISM: general

(ISM:) HII regions

(ISM:) Herbig-Haro objects

ISM: individual objects: . . .

(except planetary nebulae)

ISM: jets and outflows

ISM: kinematics and dynamics

ISM: lines and bands

ISM: magnetic fields

ISM: molecules

(ISM:) photodissociation region (PDR)

(ISM:) planetary nebulae: general

(ISM:) planetary nebulae: individual: ...

ISM: structure

ISM: supernova remnants
The Galaxy

Galaxy: abundances

Galaxy: bulge

Galaxy: centre

Galaxy: disc

Galaxy: evolution

Galaxy: formation

Galaxy: fundamental parameters

Galaxy: general

(Galaxy:) globular clusters: general

(Galaxy:) globular clusters: individual: ...

Galaxy: halo

Galaxy: kinematics and dynamics

(Galaxy:) local interstellar matter

Galaxy: nucleus

(Galaxy:) open clusters and associations: general

(Galaxy:) open clusters and associations: individual: ...

(Galaxy:) solar neighbourhood

Galaxy: stellar content

Galaxy: structure

\section{Galaxies}

galaxies: abundances

galaxies: active

galaxies: bar

(galaxies:) BL Lacertae objects: general

(galaxies:) BL Lacertae objects: individual: ...

galaxies: bulges

galaxies: clusters: general

galaxies: clusters: individual: ...

galaxies: clusters: intracluster medium

galaxies: disc

galaxies: distances and redshifts

galaxies: dwarf

galaxies: elliptical and lenticular, cD

galaxies: evolution

galaxies: formation

galaxies: fundamental parameters

galaxies: general

galaxies: groups: general

galaxies: groups: individual: ...

galaxies: haloes

galaxies: high-redshift

galaxies: individual: . . .

galaxies: interactions

(galaxies:) intergalactic medium

galaxies: irregular

galaxies: ISM

galaxies: jets

galaxies: kinematics and dynamics

(galaxies:) Local Group

galaxies: luminosity function, mass function

(galaxies:) Magellanic Clouds

galaxies: magnetic fields

galaxies: nuclei

galaxies: peculiar

galaxies: photometry

(galaxies:) quasars: absorption lines

(galaxies:) quasars: emission lines

(galaxies:) quasars: general 
(galaxies:) quasars: individual: ... .

(galaxies:) quasars: supermassive black holes galaxies: Seyfert

galaxies: spiral

galaxies: starburst

galaxies: star clusters: general

galaxies: star clusters: individual: . . .

galaxies: star formation

galaxies: statistics

galaxies: stellar content

galaxies: structure

\section{Cosmology}

(cosmology:) cosmic background radiation

(cosmology:) cosmological parameters

(cosmology:) dark ages, reionization, first stars

(cosmology:) dark energy

(cosmology:) dark matter

(cosmology:) diffuse radiation

(cosmology:) distance scale

(cosmology:) early Universe

(cosmology:) inflation

(cosmology:) large-scale structure of Universe

cosmology: miscellaneous

cosmology: observations

(cosmology:) primordial nucleosynthesis

cosmology: theory

\section{Resolved and unresolved sources as a function of wavelength}

gamma-rays: diffuse background

gamma-rays: galaxies

gamma-rays: galaxies: clusters

gamma-rays: general

gamma-rays: ISM

gamma-rays: stars

infrared: diffuse background

infrared: galaxies

infrared: general

infrared: ISM

infrared: planetary systems

infrared: stars

radio continuum: galaxies

radio continuum: general

radio continuum: ISM

radio continuum: planetary systems

radio continuum: stars

radio continuum: transients

radio lines: galaxies

radio lines: general

radio lines: ISM

radio lines: planetary systems

radio lines: stars

submillimetre: diffuse background

submillimetre: galaxies

submillimetre: general

submillimetre: ISM

submillimetre: planetary systems

submillimetre: stars

ultraviolet: galaxies ultraviolet: general

ultraviolet: ISM

ultraviolet: planetary systems

ultraviolet: stars

$\mathrm{X}$-rays: binaries

$\mathrm{X}$-rays: bursts

X-rays: diffuse background

$\mathrm{X}$-rays: galaxies

$\mathrm{X}$-rays: galaxies: clusters

X-rays: general

X-rays: individual: ... .

X-rays: ISM

X-rays: stars

Transients

(transients:) black hole mergers

(transients:) black hole - neutron star mergers

(transients:) fast radio bursts

(transients:) gamma-ray bursts

(transients:) neutron star mergers

transients: novae

transients: supernovae

transients: tidal disruption events 